\title{
PRICE DISCRIMINATION AND THE LOCATION CHOICE OF A DURABLE GOODS MONOPOLY
}

\section{PAOLO GARELLA}

SETTEMBRE 1997 


\section{Introduction}

The traditional analysis of the location choice by a monopoly has been developed thinking of static monopolies, namely monopolies selling non-durable goods. At the same time, the spatial approach has been widely used in applications to the choice of product design. In a spatial context, the question is if monopoly power leads to the plant location which minimizes transport costs. In terms of product characteristics the question is whether a monopoly will produce the variety which maximizes the social welfare-I do not treat here the case of multiplant and multiproduct monopoly. The definition of product specification by way of spatial models, following Hotelling (1931), is usually one of horizontal differentiation, where the consumers do not unanimously rank the different specifications of a good footnote .

In the present paper I shall make use of the definition of a "spatial network", which is familiar to students in spatial economics. Broadly speaking a location network is a set of interconnected market points. The location of a seller of non-durables on a network has been extensively studied and it is known to be socially inefficient when mill pricing is used and socially efficient when delivered pricing is used-see Gabszewicz and Thisse (1986). Under mill pricing the consumers bear the transportation cost. However, under delivered pricing the monopolist directly bears the transportation costs. This is why the seller chooses the location on the network which is socially optimal—for the quantities he decides to sell footnote. The question in the present paper is whether a monopolist who sells a durable good will locate in the same way as predicted by the theory for standard monopolies.

Although the literature on durable goods monopoly is rather large, it has not considered so far the location problem. A summary of this literature is beyond the scope of the present paper; however, it is worth recalling some of its fundamental features. In a deterministic context it is well understood that the durable good differs from the static (non-durable) monopoly only if the seller cannot commit to a sequence of prices over time. This absence of commitment creates an incentive to reduce prices in the future; the reason is that buyers who have bought in the past do not re-enter the market so that the monopoly can sell only to consumers with lower and lower valuation for the good. This is known to be harmful to the monopolist, and I shall refer to this situation as to the 'Coase problem' from Coase (1972). It can be shown that the monopolist intertemporal profits in the absence of commitment are lower than they could be under full commitment. The gist of the argument is that consumers will correctly anticipate the price reductions and decide the date of purchase to their convenience. The monopolist then is in competition with its future self, as today demand depends upon tomorrow prices.

Several ways to escape from the Coase problem by gaining commitment on future prices have been identified in the literature. For instance, renting instead of selling is one such escape (see Bulow (1982)); reducing the durability (Bulow (1986)); capacity limits (De Graba (1995)), or rising marginal cost functions (Kahn (1986)); rationing demand is another possibility (Denicolò and Garella (1996)). I shall show below that locating at some distance from the market nodes on a spatial network is also a way to escape from the Coase problem. Indeed, by locating at a sufficient distance from a given market node, the monopolist creates a lower bound for his future price on that node, exploiting the existence of transport costs. This is a way to buy some commitment on future prices. The need to gain commitment then, as it is shown below, may lead the monopolist away from the socially optimal location on a location network with several market nodes.

The result of inefficient location is obtained in two different examples; in both the location network displays two market nodes connected by an arc. The time horizon is of two periods. In the first example (in Section 3 below) the presence of two market nodes is not essential to the argument which, instead, rests upon the property that the inverse demand function be discontinuous. The driving force is represented there by the commitment not to serve in the second period low valuation consumers on one of the two market nodes by locating at a sufficient distance from it. The monopolist who locates at the profit maximizing spot manages to sell only at the first period; nevertheless, the location choice depends upon the discount 
parameter. By contrast the socially optimal location does not depend upon discounting. The monopoly location, furthermore, is not on a vertex of the network, a feature that breaks the correspondence between the monopoly preferred location and the "Hakimi Theorem", from Hakimi (1964).

In the second example (Section 4 below) demand is continuous and smooth and at the equilibrium the monopoly makes sales at both periods. The presence of more than one market node is crucial in this second set-up. By increasing the distance from a given node the monopolist who price-discriminate (delivered pricing) increases the cost to bring a unit to destination. This increase in unit costs could never be optimal if there were only one market node (reducing the model to the standard non-spatial approach), in fact a non-spatial monopoly, with a smooth demand, does not find it convenient an increase in costs footnote. Here by contrast the location choice affords a way to manipulate the second period prices on both market nodes and, thereby, the demand functions at the first and second period.

One can apply the results to a model of product differentiation, after reinterpreting the geographical space as a space of product characteristics. The two market nodes here represent two alternative specifications of the good. Obviously, more general distributions of consumers over the characteristics space could be considered and the present paper in this respect only represents a cursory introduction to the topic. Mill pricing is more appropriate to the product selection interpretation. A sketchy analysis of mill pricing is contained in Section 5. Quite immediately, the result obtained for the spatial interpretation carries on, implying that the monopolist choice can be inefficient. Section 6 concludes the paper.

\section{Location on a Network}

Define footnote a network $N$ as a subset of $\Re^{2}$, where $N$ is the union of a finite number of arcs, of a well defined length. Each arc in $N$ intersects at least one and at most two other arcs in $N$. Arcs can intersect each other only at their extremities. The set of vertices, $V$, of the network is made of the extremities of the arcs. $S$ denotes a subset of $V$ with typical element $s^{i}$, for $i=1, \ldots, m$. At each point $s^{i} \in S$-and only at such points-is associated a number $p_{i}\left(\right.$ in $\left.\Re^{+}\right)$ and a function $D_{i}\left(p_{i}\right)$ from $\mathfrak{R}^{+}$to itself. Consider the problem known as the Weber problem-from Weber (1909): Choose the location $s$ in $N$ which minimizes the function

$$
T(s)=\sum_{i=1}^{m} t D_{i}\left(p_{i}^{*}\right) d\left(s, s^{i}\right),
$$

where $t$ is a positive constant, the $p_{i}^{*}$ 's are given, and $d\left(s, s^{i}\right)$ is the distance (to be defined shortly) between two points, $s$ and $s^{i}$ belonging to a network. The distance between two points is defined as the length of the shortest route on $N$, linking the two points.

A result due to Hakimi (1964), simplifies the search for a solution to the Weber problem:

Theorem (Hakimi,1964): The point $s \in N$, which minimizes $T(s)$, belongs to $V$.

The Weber problem, interpreted in an economic set-up, amounts to the minimization of total transport costs incurred for transferring quantities of an output footnote to a number of markets (the points $s^{i}$ in $S$ ) located on a network $N$, for given prices $p_{i}^{*}$ at each destination in $S$. Total transport cost on each node $t d\left(s, s^{i}\right)$ is made up of the per-unit transport cost multiplied by the quantity demanded at the prevailing price $p_{i}^{*}$. From the point of view of spatial economics this is clearly a relevant problem, and the Hakimi theorem a useful result. However, the Hakimi theorem leads to other interesting corollaries from an economic viewpoint. It can be easily seen in fact that a monopolist who is allowed to practice spatial price discrimination and quoting delivered prices on each market node $s^{i}$, will choose the same location $s^{*}$ which minimizing transport costs is also socially optimal. In fact, consider a monopolist who produces at costs $c(q)=c\left(\sum D\left(p_{i}^{*}\right)\right)$; his profit maximization program shall then be written as

$$
\max _{p_{i}, s} \sum_{i=1}^{m} D_{i}(p) p_{i}-c\left(\sum D\left(p_{i}\right)\right)-\sum_{i=1}^{m} t D_{i}\left(p_{i}\right) d\left(s, s^{i}\right) .
$$

It can be shown that the price solution $\left(p_{i}^{*}\right)_{i=1, \ldots, m}$ determines the quantities $D_{i}\left(p_{i}^{*}\right)$ on each 
market, and the maximization of profits implies the minimization of $T(s)$ for those quantities with respect to $s$. Note again that to have the monopolist choice of $s$ coincide with the socially optimal $s$ for given quantities, it must be possible to practice perfect spatial price discrimination.

Proposition A monopolist selling from a single outlet on $N$ and practicing perfect price discrimination on the different market nodes will locate his outlet at the location which minimizes total transport costs $T(s)$. This location belongs to $V$.

\section{Durable Goods}

Consider the following example of a monopolist selling a durable good to a population of consumers on the network to be described shortly. The time horizon consists of two periods and unit production costs are assumed to be zero to simplify the algebra. The monopolist can sell at the beginning of periods 1 and 2, and a consumer who has bought at period 1 has zero demand for the good at period 2 (durability). Each consumer buys at most one unit of the good. The possibility of renting is excluded and the monopolist cannot commit to future prices.

Assume the network $N$ to be made of two market nodes connected by a segment of length $\ell<1$. Market node $\mathrm{A}$ is located at end-point 0 and node $\mathrm{B}$ at endpoint $\ell$ (in the notation of Section $2, s^{A}=0$ and $s^{B}=\ell$ ). The cost of carrying one unit of the good over the distance $d\left(s, s^{i}\right)$ is equal to $\sqrt{\left(s-s^{i}\right)^{2}}$, for $i=A, B$.

The demand function in each market is a discontinuous function, as it shall be clear from the description below. In market node A there are "high valuation" consumers, i.e. those with valuation of the good given by $v$, with $v$ distributed over the interval $[1,1.5]$ according to the uniform function $F(v)=\frac{v-1}{(0.5)}$. In addition to these buyers, at market node A there is a mass $x$ of low valuation buyers, who value the good at $v_{0}$, with $v_{0}<1$. Assume also

A.1.

$v_{0}<\ell / 2$. $v^{B}=1$

In node $\mathrm{B}$ there are only a measure $B$ of consumers with unanimous valuation for the good

The utility function of consumer with valuation equal to $v$ and located in $s^{i}$ is given by zero if he does not buy, while buying a unit at date $t$ gives utility

$$
u=\delta^{t-1}\left(v-p^{i}\right) \quad \text { for } t=1,2 ; \quad \text { and } \quad i=A, B .
$$

Different markets discount the future at the same rate, $\delta$, as the monopolist. Assume further that

A.2.

$$
v_{0}(x+A)<A \text {. }
$$

The location chosen by the monopolist is denoted by $s$, with $s \in[0, \ell]$. Let define the static demand and profit as those which apply when there is no second period (or, which is equivalent, when the monopolist can commit to a constant price over the two periods).

Definition The static monopoly demand on node $\mathrm{A}$ is $D(p)=\min \{A, 2[(3 / 2)-p] A\}$ for $v_{0}<p<3 / 2, D(p)=A+x$ for $v_{0} \geq p$, and $D(p)=0$ otherwise. The static monopoly profit on node A when $\mathrm{s}=0$ is $p^{A} D^{A}\left(p^{A}\right) \equiv \bar{\pi}^{A}$.

The reason for disregarding the static profit for locations different from $s=0$ is that the profit $\bar{\pi}^{A}$ is sufficient as a reference point, as it shall become apparent in the sequel.

Assumption (A.2) implies that the static profit made by the monopolist on node A is larger if he sells at price 1 only to the high types rather than selling to everybody at price $v_{0}$. Furthermore, it is easy to see that the price which maximizes the function $p[(3 / 2)-p] 2 A$ is lower than 1 (in particular it is equal to $3 / 4$ ) so that $p_{1}^{*}=1$ is the price which maximizes the static local profit on A. This is the way in which the Coase argument shows up in the present example; the monopolist located at $s=0$ would like to commit to a price sequence $p_{1}^{A}=p_{2}^{A}=1$ and realize the static monopoly profits $\bar{\pi}^{A}=A$. However, once all the high valuation consumers have bought in the first period there is no way to restrain the monopolist from lowering the second period price to $v_{0}$ 
and serve the low valuation consumers. But then the high valuation consumers may not buy in the first period.

Here, rational expectations are assumed to characterize the expectation formation by consumers, so that a price path is time consistent only if the buyers correctly anticipate the second period price.

If the monopolist cannot commit to a price sequence then it is well known that his profit cannot exceed the static monopoly profit footnote .

Obviously, the static demand in B is rectangular with $D(p)=B$ if $p \leq 1$ and $D(p)=0$ otherwise. Clearly, $p^{*}=1$ is the static optimal delivered price on market $\mathrm{B}$ if the monopolist locates in B. There is no "Coase problem" on node B in this example.

To develop the argument leading to the result I shall analyze first the price sequence for location $s=0$, then the price sequence for locations $0<s<v_{0}$, and finally that for $s \geq v_{0}$. The maximum profit associated to each location choice is computed at each step and then a comparison will show that under some conditions on the parameters the best location is one to the right of $v_{0}$ (in fact it is just a trifle to the right of $v_{0}$ ).

1. Consider the pricing problem of the monopolist located at point $s^{A}=0$. Clearly, if $p_{1}>v_{0}$ the monopolist has an incentive to sell at the second period to consumers who have not bought in the first period, at a price which shall be lower than the first period price. Then, since consumers can wait, the consumer $v$ of node A at the first period buys only if the price $p_{1}^{A}$ is such that

$$
v-p_{1}^{A} \geq \delta\left(v-p_{2}^{A}\right) .
$$

So that given $p_{1}^{A}>v_{0}$, only the types with valuation larger than $\tilde{v} \equiv \frac{p_{1}^{A}-\delta \check{p}_{2}^{A}}{(1-\delta)}$ shall buy at the first period, where $\breve{p}_{2}^{A}$ is the second period price anticipated by the buyers.

To proceed, first it must be shown that $p_{2}^{A}$ cannot be set higher than 1 along a time consistent price path, and then it shall be easy to pin down the equilibrium price sequence for $s=0$. Since the second period demand, for $p_{2}^{A}>1$, is the measure of buyers with valuations in the interval $\left[p_{2}^{A}, \tilde{v}\right]$, then it is given by $2 A\left(\tilde{v}-p_{2}^{A}\right)$. Therefore, to have $p_{2}^{A} \geq 1 p_{2}^{A}$ must be the solution to

$$
\max _{p_{2}} 2 A\left(\tilde{v}-p_{2}\right)\left(p_{2}-s\right) \text {. }
$$

Since for $s=0$, this price is lower than 1, as shown in the proof of the following Lemma, it is possible to state:

Lemma If $s=0$, the only price sequence $\left(p_{1}^{A}, p_{2}^{A}\right)$ which is time consistent is with

$$
p_{1}^{A}=(1-\delta) v_{0}+\delta v_{0} \text { and } p_{2}^{A}=v_{0} \text {. }
$$

Proof.

(a) $p_{2}^{A} \geq 1$ cannot be part of a time consistent price path. Indeed, assume $p_{2} \geq 1 . p_{2}>p_{1}$ cannot be a time consistent price sequence. If $p_{2}<p_{1}$ only consumers with valuation higher than $\tilde{v} \equiv \frac{p_{1}-\delta p_{2}}{(1-\delta)}$ shall buy at the first period. Assume then $1<p_{2}<p_{1}<3 / 2$. The second period price is then

$$
\arg \max _{p_{2}} 2 A\left(\frac{p_{1}-p_{2}}{1-\delta}\right) p_{2}=\frac{p_{1}}{2}
$$

And the second period profits are $\frac{2 A}{(1-\delta)}\left(\frac{p_{1}}{2}\right)^{2}$.

The first period demand, if $1<p_{2}<p_{1}<3 / 2$, shall then be equal to $2 A\left(\frac{3}{2}-\tilde{v}\right)$ which is equal to $2 A\left[\frac{3}{2}-\frac{2 p_{1}-\delta p_{1}}{2(1-\delta)}\right]$. The price at the first period shall be set equal to

$$
\arg \max _{p_{1}} p_{1} 2 A\left(\frac{3}{2}-\frac{2 p_{1}-\delta p_{1}}{2(1-\delta)}\right)+\delta\left[\frac{2 A}{(1-\delta)}\left(\frac{p_{1}}{2}\right)^{2}\right]=\frac{3(1-\delta)}{4-3 \delta} .
$$

This value of $p_{1}$ is lower than 1 for all values of $\delta \in[0,1]$, but this contradicts that the second period demand be made of high valuation types with $p_{2}^{A}>1$.

(b) $1 \geq p_{2}>v_{0}$ cannot be part of a time consistent price path. Indeed, since demand in A is 
totally inelastic over the range of prices $\left(v_{0}, 1\right]$, then either it is $p_{2}=1$, which contradicts part (a) above, or $p_{2} \leq v_{0}$.

(c) Since demand is equal to $A+x$ for all prices lower than $v_{0}$, then the only second period price which can be part of a time consistent price path is $p_{2}^{A}=v_{0}$. It follows that first period demand, $D_{1}^{A}\left(p_{1}^{A}, v_{0}\right)$, for $p_{1}$ in the range $[1,3 / 2]$ shall be given by $D_{1}^{A}\left(p_{1}^{A}, v_{0}\right)=$ $\min \left\{A\left[3-\frac{2\left(p_{1}-\delta v_{0}\right)}{(1-\delta)}\right], A\right\}$. The function,

$$
A\left[3-\frac{2\left(p-\delta v_{0}\right)}{(1-\delta)}\right] p
$$

however, is maximized with respect to $p$ at $p_{1}^{A}=\left(\frac{3-\delta\left(3-4 v_{0}\right)}{4}\right)$ which in turn is lower than 1 (given that $v_{0}<\ell / 2$ and $\ell<1$ ). But then the best first period price is the price which makes the buyer with willingness to pay exactly equal to 1 indifferent between buying soon or waiting for $p_{2}=v_{0}$. This price is the solution to $1-p_{1}=\delta\left(1-v_{0}\right)$ and it indeed corresponds to $p_{1}=(1-\delta)+\delta v_{0}$, which, given $v_{0}<\ell / 2<1$, lies as required in the interval $\left(v_{0}, 1\right)$. [End Proof]

The highest profit that the monopolist can make if he locates in $s=0$ is therefore, the one corresponding to delivered prices $p_{1}^{B}=p_{2}^{B}=1$, and $p_{1}^{A}=(1-\delta)+\delta v_{0}, p_{2}^{A}=v_{0}$.

Total profits for $s=0$ are

$$
\pi(0) \equiv A\left[(1-\delta)+\delta v_{0}\right]+\delta v_{0} x+B-\ell B .
$$

2. Consider now any location in the interval $\left[0, v_{0}\right)$. Since the unit transport cost to market $A$ is less than $v_{0}$ the monopolist cannot commit to refuse to sell to customers with valuation $v_{0}$ at the second period. On the other hand, a second period price such that $v_{0}<p_{2} \leq 1$ is not time consistent given that demand on node $\mathrm{A}$ is totally inelastic over that range of prices.

Furthermore, a second period price larger than 1 implies that $p_{2}^{A}=\arg \max _{p_{2}}\left(p_{2}-s\right) 2 A\left(\tilde{v}-p_{2}\right)$, namely $p_{2}^{A}=\frac{p_{1}+s}{2}$. This implies a second period profit equal to $\frac{A}{2(1-\delta)}\left(p_{1}^{A}-s\right)^{2}$. Then the first period price should solve

$$
\max _{p_{1}}\left(p_{1}-s\right) 2 A\left(\frac{3}{2}-\frac{2 p_{1}-\delta p_{1}}{2(1-\delta)}\right)+\delta\left[\frac{A}{2(1-\delta)}\left(p_{1}-s\right)^{2}\right] .
$$

One can find that the solution to ( ref: MS ) is

$$
p_{1}^{A}=\frac{3(1-\delta)+s(2-3 \delta)}{4-3 \delta},
$$

which, again, is lower than 1 for all values of $\delta$. But this contradicts that $p_{2}>1$. In this case also, the only time consistent price sequence is $p_{2}^{A}=v_{0}$ and $p_{1}^{A}=(1-\delta)+\delta v_{0}$. The prices are the same as for the location $s=0$, but transport costs are positive, therefore profits on A are lower.

Total profits for $s \in\left(0, v_{0}\right)$ are

$$
A\left[(1-\delta)+\delta v_{0}-s\right]+\delta\left(v_{0}-s\right) x+B-(l-s) B .
$$

3. Consider location $s>v_{0}$. At the second period it is impossible to serve the low valuation consumers without incurring a loss since the unit transport cost from $s$ to 0 is larger than the reservation price $v_{0}$. Then it is credible to refuse selling to low valuation customers by maintaining $p_{2}^{A}=1$. The price sequence $p_{1}^{A}=p_{2}^{A}=1$ is indeed time consistent.

Total profits from location $s>v_{0}$ are

$$
A(1-s)+B-(\ell-s) B .
$$

4. A comparison can now be made. The discussion so far implies that any location in the interval $0<s \leq v_{0}$, implies, as argued above, that the second period price be low enough to serve the low valuation buyers in node A. Then, the profit from these locations is easily seen to 
be lower than profit $\pi(0)$ in equation ( ref: P0 ). It is sufficient then to compare ( ref: PSS ) with ( ref: P0 ). It is immediate to see, that a location $s>v_{0}$ dominates $s=0$ if

$$
-s(A-B)+\delta A-\delta v_{0}(A+x) \geq 0 .
$$

Remark that the L.H.S. in this inequality is increasing in $\delta$ : The importance attributed to the future makes commitment more valuable. For any value of $\delta$, furthermore, this inequality strictly holds for $A=B$, under assumption (A.2). The monopolist therefore shall locate at a point $v_{0}+\epsilon$, whenever $A=B$, but also when $A$ is smaller than $B$ and condition ( ref: C ) holds. Profits from locating close to $v_{0}$ are (neglecting $\epsilon$ )

$$
A\left(1-v_{0}\right)+B-\left(\ell-v_{0}\right) B .
$$

Total sales by the monopolist located at $v_{0}+\epsilon$ are $A+B$. By contrast the socially optimal location, given sales $A+B$ is $s=0$ if $A>B$ and $s=\ell$ if $B>A$, for all values of $\delta$. Here $\delta$ may play a crucial role in directing the monopolist choice, while it plays no role in the decision about which is the socially optimal location.

Proposition If inequality ( ref: $\mathrm{C}$ ) holds, the location choice of a durable goods monopolist is socially inefficient.

Also,

Remark If inequality ( ref: $\mathrm{C}$ ) holds, the location choice of the monopolist in the example does not belong to a vertex of the network $N$.

Remark The location choice of a durable goods monopolist is affected by the time discount factor.

The first Remark means that the Hakimi theorem is of no help in the case of a durable goods monopoly.

As a comment to the second Remark, note that if all sales are made at the first period the socially optimal location is not affected by the rate of time preferences. Next Section considers a case where sales are made at both periods.

\section{Smooth Demand Functions and Discounting}

\section{Manipulating Demand through the Choice of Location}

To see that the example in section 3 highlights a more general phenomenon, consider the same location Network, but assume that at each market node there is a smooth distribution of willingness to pay. In particular, let the valuation of buyers be distributed over the interval $[0,1]$ at both nodes, with $F(v)=v^{\alpha}$ at node A, and $F(v)=v^{\beta}$ on node $\mathrm{B}$, where $\alpha$ and $\beta$ are nonnegative scalars. Then it can be seen that the second period demand is given on each node by the measure of the buyers with valuation comprised in the interval $\left[\tilde{v}, p_{2}^{i}\right]$ for $i=A, B$. The measure of this quantity is $\tilde{v}^{\alpha}-\left(p_{2}^{A}\right)^{\alpha}$ on market A and $\tilde{v}^{\beta}-\left(p_{2}^{B}\right)^{\beta}$ on market B. Similarly, first period demand on node A (resp. B) is the quantity $1-\tilde{v}^{\alpha}$ (resp. $\left.1-\tilde{v}^{\beta}\right)$.

Let $p_{2}^{A}\left(p_{1}^{A}, s\right),\left(\right.$ resp. $\left.p_{2}^{B}\left(p_{1}^{B}, s\right)\right)$ denote the second period price which solves the problem

$$
\max _{p}(p-s)\left(\tilde{v}^{\alpha}-p^{\alpha}\right)
$$

$\left(\right.$ resp. $\left.\max _{p}(p-s)\left(\tilde{v}^{\beta}-p^{\beta}\right)\right)$.

Then the valuation of the consumer on node $\mathrm{A}$ that is indifferent between buying at period 1 or waiting for the second period price is

$$
\tilde{v}^{A}\left(p_{1}^{A}, s\right)=\frac{p_{1}^{A}-\delta p_{2}^{A}\left(p_{1}^{A}, s\right)}{(1-\delta)}
$$

Let $\tilde{v}^{A}\left(p_{1}^{A}, s\right) \equiv v_{A}$, to simplify notation. Respectively, it is $\tilde{v}^{B}\left(p_{1}^{B}, s\right)=\frac{p_{1}^{B}-\delta p_{2}^{B}\left(p_{1}^{B}, s\right)}{(1-\delta)} \equiv v_{B}$.

Define $\pi_{1}^{A}=\left(p_{1}^{A}-s\right)\left(1-\left(v_{A}\right)^{\alpha}\right)$, and $\left.\pi_{2}^{A}=\left[p_{2}^{A}\left(p_{1}^{A}, s\right)\right)-s\right]\left[\left(v_{A}\right)^{\alpha}-\left(p_{2}^{A}\left(p_{1}^{A}, s\right)\right)^{\alpha}\right]$, then total intertemporal profits on node $A$, (resp. B) discounted at period 1 are

$$
\pi^{A}=\pi_{1}^{A}+\delta \pi_{2}^{A},
$$

and respectively, letting $\pi_{1}^{B}=\left[p_{1}^{B}-(\ell-s)\right]\left[1-\left(v_{B}\right)^{\beta}\right]$ and 
$\left.\pi_{2}^{B}=\delta\left[p_{2}^{B}\left(p_{1}^{B}, s\right)\right)-(\ell-s)\right]\left[\left(v_{B}\right)^{\beta}-\left(p_{2}^{B}\left(p_{1}^{B}\right)\right)^{\beta}\right]$, total intertemporal profits on node B are $\pi^{B}=\pi_{1}^{B}+\delta \pi_{2}^{B}$

The choice of $p_{1}^{A}$ (resp. $p_{1}^{B}$ ) is made so as to maximize profits on both nodes for any location $s$. This maximization affords a maximum denoted by

$\Pi=\Pi^{A}+\Pi^{B}=\Pi_{1}^{A}+\Pi_{1}^{B}+\delta\left(\Pi_{2}^{A}+\Pi_{2}^{B}\right)$.Using the envelope theorem and the notation $v_{i}^{\prime}=\frac{\partial v_{i}\left(p^{i}, s\right)}{\partial s}$ for $i=A, B$, one finds that the derivative with respect to $s$ of the profit function on node $\mathrm{A}$, when $p_{1}^{A}$ is chosen to maximize profits is the sum of two terms:

$$
\frac{\partial \Pi^{A}}{\partial s}=\Pi_{A 1}^{\prime}+\delta \Pi_{A 2}^{\prime}
$$

with

$$
\Pi_{A 1}^{\prime}=-\left(1-v_{A}^{\alpha}\right)+s \alpha\left[v_{A}^{\alpha-1}\right] v_{A}^{\prime},
$$

and

$$
\Pi_{A 2}^{\prime}=\left[\frac{\partial p_{2}^{A}}{\partial s}-1\right]\left[v_{A}^{\alpha}-\left(p_{2}^{A}\right)^{\alpha}\right]+\left(p_{2}^{A}-s\right) \alpha\left[v_{A}^{\alpha-1} v_{A}^{\prime}-\left(p_{2}^{A}\right)^{\alpha-1} \frac{\partial p_{2}^{A}}{\partial s}\right]
$$

As the notation suggests, ( ref: pia1 ) and ( ref: pia2 ) are the derivatives of the first and second period components of the sum of discounted profits respectively.

As $\Pi_{A 1}^{\prime}+\delta \Pi_{A 2}^{\prime}$ is the derivative of profits on node $\mathrm{A}$ so $\Pi_{B 1}^{\prime}+\delta \Pi_{B 1}^{\prime}$ is the total derivative footnote of the sum of discounted profits on node $\mathrm{B}$. The total derivative of profits is of course $\Pi_{A 1}^{\prime}+\Pi_{B 1}^{\prime}+\delta\left(\Pi_{A 2}^{\prime}+\Pi_{B 2}^{\prime}\right)$.

One can note that in the total derivative are present four "quantity components" these are the terms $-\left(1-v_{A}^{\alpha}\right)$ and $-\delta\left(v_{A}^{\alpha}-\left(p_{2}^{A}\right)^{\alpha}\right)$ for node $\mathrm{A}$, and $\left(1-v_{B}^{\beta}\right)$ and $\delta\left(v_{B}^{\beta}-\left(p_{2}^{B}\right)^{\beta}\right)$ for node B. Besides these "quantity components" there appear other terms reflecting the property that by changing the second period price the values for $v_{A}$ and $v_{B}$, which enter the demand function at date 1 and 2, change. In particular, this follows from the property that the valuation which renders a consumer indifferent between buying at date 1 or waiting depends on $p_{2}\left(p_{1}, s\right)$.

The monopolist changing his location modifies not only the current and future prices, but also on each node the intercept on the price axis of the second period demand $\left(v_{A}\right.$ and $\left.v_{B}\right)$, and the intercept of the first period demand (for example for $\alpha=1$ this intercept on node A is $\left.1+\frac{p_{2}\left(p_{1}, s\right)}{(1-\delta)}\right)$.

Finally there appear a term for each node accounting for the effect of a change in $s$ on the second period profits through a change in the second period price, $\frac{\partial p_{A}^{2}}{\partial s}\left[\left(v_{A}^{\alpha}-\left(p_{2}^{A}\right)^{\alpha}\right]\right.$ and $\frac{\partial p_{B}^{2}}{\partial s}\left[\left(v_{B}^{\beta}-p_{2}^{B}\right)^{\beta}\right]$.

Consider now the problem from a social point of view. The comparison now takes an intertemporal dimension which was absent in the previous section example since there the monopolist sold only at the first period. The comparison involves delivery of the good at the same dates and to the same consumers as in the monopoly solution (a discount of time preferences must be applied to future deliveries). This way, total utility from consumption of the good is kept constant. The location which minimizes transport costs for the quantities sold by the monopolist is the one which solves

$$
\min _{s} T(s, \delta)=s\left(1-v_{A}^{\alpha}\right)+s \delta\left[v_{A}^{\alpha}-\left(p_{2}^{A}\right)^{\alpha}\right]+(l-s)\left\{\left(1-v_{B}^{\beta}\right)+\delta\left[v_{B}^{\beta}-\left(p_{2}^{B}\right)^{\beta}\right]\right\} .
$$

The first derivative with respect to $s$ of the objective function is

$$
\left(1-v_{A}^{\alpha}\right)+\delta\left[v_{A}^{\alpha}-\left(p_{2}^{A}\right)^{\alpha}\right]-\left(1-v_{B}^{\beta}\right)-\delta\left[v_{B}^{\beta}-\left(p_{2}^{B}\right)^{\beta}\right] .
$$

Clearly, ( ref: foc 2 ) only involves the "quantity terms", while the effects of location on the consumers' behavior (the changes in the values for the $v^{\prime} s$ ) are missing. Therefore, since 
( ref: foc2 ) does not depend on $s$, the solution is at one of the market nodes (the Hakimi Theorem holds for the social optimum). This shows again that the two solutions, the one for the monopolist location, and the socially optimal one, may differ.

\section{An Example}

The expressions for the derivative of total profits with respect to $s$ are quite complex functions. The calculations become rather easy, however in a case which can be used as an illustrative example. Let again describe the node B as one characterized by a rectangular demand: that is, the population of consumers in B is formed of identical consumers who value the good at $v=1$. Let the mass of these consumers be equal to 1 . Furthermore, let market node $\mathrm{A}$ be populated by consumers with valuation in $[0,1]$, and let $\alpha=1$. Then it can be easily seen that $v_{A}=\left(p_{1}-\delta p_{2}\right) /(1-\delta)$, the price $p_{2}^{A}$ which maximizes second period profits is $p_{2}^{A}\left(p_{1}^{A}\right)=\left(p_{1}^{A}+s\right) / 2$. Plugging in this value of $p_{2}^{A}$ into the profit function for node $\mathrm{A}$

$$
\pi_{A}=\left(p_{1}^{A}-s\right)\left(1-v_{A}\right)+\delta\left(p_{2}^{A}-s\right)\left(v_{A}-p_{2}^{A}\right),
$$

and maximizing with respect to $p_{1}^{A}$, yields

$$
p_{1}^{A}=(1+s)(1-\delta) / 2 \quad p_{2}^{A}=(1+s)(1-\delta) / 4+s / 2 .
$$

Quite obviously, on node B one has that $p_{1}^{B}=p_{2}^{B}=1$ and all consumers there buy at date 1 .

Then, letting $p^{n}=\left(p_{1}^{A}-s\right)=\frac{(1-\delta)-s(2-\delta)}{2}$, total discounted profits for the monopolist are

$$
p^{n}\left[1-\frac{(1-s)(2-\delta)}{2}+\frac{s \delta}{2(1-\delta)}\right]+\frac{\delta}{2(1-\delta)}\left(p^{n}\right)^{2}+1-(l-s) .
$$

This is a concave function of $s$, and for the value of $\delta=0.8$ and $\ell=0.6$ it is displayed in the graph in Figure-1. Clearly the monopolist optimal location is on an interior point of the segment connecting the two market nodes.

\section{Mill Pricing and Horizontal Differentiation}

The product characteristics interpretation of the location network with two market nodes is possible if one thinks of a rather special distribution of consumers tastes or if one considers the case of buyers that can be grouped into two categories - men and women, or young and old-who prefer alternative specifications of a good. More general distributions of consumers tastes are out of the scope of the present paper footnote. In any case it is worthwhile briefly considering the location choice under mill pricing.

That mill pricing leads to inefficient locations is already well known. For instance, Gabszewicz and Thisse (1986a) refer to an example with a network similar to the one in Section 4 above, with a demand function given by $\max \left\{0, \alpha-\left(p+t\left(s^{i}, s\right)\right)^{2}\right\}$ on each node, where $\alpha$ is a positive constant. There, a location at the center of the arc connecting the two market nodes is optimal for sufficiently large values of the parameter $\alpha$.

The results obtained in Section 3 above extend to the case of mill pricing. This case implies 
that consumers bear the transport cost and that the monopolist quotes a uniform price to all customers. The utility from purchase of a buyer in node A, given a mill price at period $t, p_{t}$ say, is equal to

$$
\delta^{t-1}\left(v-p_{t}-s\right) \quad \text { for } \quad t=1,2 .
$$

The utility for buyers located at node $\mathrm{B}$ is

$$
\delta^{t-1}\left[v-p_{t}-(\ell-s)\right] .
$$

Then, the indifference between waiting and buying soon obtains for

$$
\hat{v}=\frac{p_{1}-\delta p_{2}}{(1-\delta)}+s
$$

Consider the demand functions described in Section 3. The presence of low valuation customers, at Node A, with valuation $v_{0}$, makes it impossible for the monopolist located at $s=0$ to use a mill price sequence like $p_{1}=1$ and $p_{2}=1$. As for the case of section 3 , the incentive to serve low valuation customers cannot be eliminated if the seller locates at (or close to) $s=0$. Again the exercise consists in comparing the maximum profits at locations $s<v_{0}$, and $s \geq v_{0}$. It is not worth repeating the analysis here as nothing new is gained from it.

\section{Conclusions}

The literature on durable goods monopoly has so far neglected the location problems, and at the same time the literature on location and product specification has not been concerned with durable goods monopolies. The present paper shows that a price discriminating monopolist will not necessarily choose a socially optimal location. The reason why is that by manipulating the transport cost the seller can commit not to lower too much the price in the future on the farthest nodes from its location. This ameliorates the profits on those nodes and, if these nodes are important enough, total profits can be increased by such a location policy. Possible applications of the theory under delivered pricing include the location of a warehouse for intermediate goods which a seller delivers to different users, or the location of a service provider whose service has the character of a durable good (like a carpenter, or a plumber who charge the customer for the traveling cost and transport of materials). As for the horizontal differentiation interpretation, one may think of the design of a machine or other investment good: this can be chosen in such a way as to reduce its usefulness for potential buyers who have a low valuation for it (for instance these buyers may dislike some features that the seller can deliberately incorporate into the good). Third degree price discrimination is implied here by the delivered price model, whether on geographic space or on consumer groups, and non discriminatory pricing applies to the mill price model.

The long established literature on the welfare effects of third degree price discrimination footnote has clarified that it may not be socially optimal to compel a monopolist to use a uniform price (either a mill price or a uniform delivered price in a spatial model) as this may result in welfare losses (recent examples are Malueg and Schwartz (1994) and Layson (1994), see also Shin, Mai and Lin (1988), while Bilas (1969) considers multiple plant firms). The welfare comparisons in the literature generally hinge upon the quantity sold on each market or upon the suppression of sales on some of the markets. Market separability (geographic or other) is clearly essential to third degree price discrimination footnote . Location effects on welfare have also been considered as shown in Anderson, de Palma and Thisse (1992), where an analysis is provided also of the number of different specifications introduced into a market.

The present paper does not consider the output effects of price discrimination, and the results only concern the location effects on welfare. Note, however, that in both the examples considered above (Section 3 and Section 4) the suboptimal location implies an increase in prices (lower output) with respect to those which maximize profits when the monopolist is set at the socially optimal location. For instance, in the example of Section 3 above, the monopolist excludes a mass of low valuation buyers by locating away from the (large) market node A. Imposing mill pricing does not necessarily lead to higher output, as it is clear from the discussion of Section 5.

The present paper leaves open some issues for further research. A major one seems to be the 
location choice of durable goods oligopolists, and the study of durable products differentiation under oligopolistic rivalry. A second issue is the location of multiplant, or multiproduct firms.

bibitem Anderson, S.; A. de Palma and J.-F. Thisse (1992): Social Surplus and Profitability under Different Spatial Pricing Policies, Southern Economic Journal, vol. 58, 934-49.

bibitem Armstrong M. and J. Vickers (1991): Welfare Effects of Price Discrimination by a Regulated Monopolist, Rand Journal of Economics, vol. 22, 571-80.

bibitem Bilas R. (1969): Third Degree Price Discrimination and the Multiple Plant Monopolist: A Note on the Allocation of Output, Southern Economic Journal, vol. 36, 82-86.

bibitem Bulow J. (1982): Durable Goods Monopolists, Journal of Political Economy, vol. 15, 314-332.

bibitem Bulow J. (1986): An Economic Theory of Planned Obsolescence, Quarterly Journal of Economics, vol. 51, 729-750.

bibitem Cheung F. K. and X. Wang (1996): Mill and Uniform Pricing: A Comparison, Journal of Regional Science, vol. 36, 129-143.

bibitem Coase R. (1972), Durability and Monopoly, Journal of Law and Economics, vol. 15, 143-149.

bibitem De Graba, P. (1995): Buying Frenzies and Seller-Induced Excess Demand, Rand Journal of Economics, vol. 26, 331-342.

bibitem Denicolò V. and P. G. Garella (1996): Rationing in a Durable Goods Monopoly, mimeo University of Bologna, Department of Economics, Strada Maggiore 45, 40123 Bologna, Italy.

bibitem Dos Santos Ferreira, R. and J.-F. Thisse (1996): Horizontal and Vertical Differentiation: The Launhardt Model, International Journal of Industrial Organization, vol. 14, 485-506.

bibitem Espinosa, M.P. (1992): Delivered Pricing, FOB Pricing, and Collusion in Spatial Markets, Rand Journal of Economics, vol. 23, 64-85.

bibitem Hanjoul P. and J.-F. Thisse (1984), The Location of a Firm on a Network, in Applied Decision Analysis and Economic Behaviour, ed. by A.J. Hughes Hallet. Den Haag: Martinus Nijoff, 1984.

bibitem Hakimi S. L. (1964), Optimum Location of Switching Centers and the Absolute Centers and Medians of a Graph, Operations Research, vol. 12, 450-459.

bibitem Hotelling, H. (1929), Stability in Competition, Economic Journal, vol. 39, 41-57.

bibitem Gabszewicz-Jaskold, J. and J.-F. Thisse (1986 a), Spatial Competition and the Location of Firms. In Location Theory. Fundamentals of Pure and Applied Economics 5, ed. by Jacques Lesourne and Hugo Sonnenschein. London, Harwood Academic Publishers, $1-71$.

bibitem Gabszewicz-Jaskold, J. and J.-F. Thisse (1986 b): On the Nature of Competition with Differentiated Products, Economic Journal, vol. 96, 160-72.

bibitem Greenhut, M. L. and H. Ohta (1979): Output Effects of Spatial Price Discrimination under Conditions of Monopoly and Competition, Southern Economic Journal, vol. 46, 71-84.

bibitem Kahn, C. (1986): The Durable Goods Monopolist and Consistency with Increasing Costs, Econometrica, vol. 54, 275-294.

bibitem Karp, L. and J. Perloff (1996), The Optimal Suppression of a Low Cost Technology by a Durable Goods Monopolist, The Rand Journal of Economics; vol. 27, 346-64.

bibitem Layson S.K. (1994): Market Opening under Third-Degree Price Discrimination, Journal of Industrial Economics, vol. 43, 335-40.

bibitem Malueg D.A. and M. Schwartz (1994): Parallel Imports, Demand Dispersion, and International Price Discrimination, Journal of International Economics, vol. 37, 167-195.

bibitem Shin, J.-j.; C.-c. Mai and J.-c. Lin (1988): A General Analysis of the Output Effects under Third Degree Price Discrimination, Economic Journal, vol. 98, 149-158.

bibitem Spulber D. F. (1989). Regulation and Markets, MIT Press, Cambridge, Mass.

bibitem Weber A. (1909), Ueber den Standort der Industrien. Tübingen: J.C.B. Mohr, 1909. English translation: The Theory of the Location of Industries. Chicago: Chicago University Press, 1929. 